\title{
COMPARAÇÃO DE MÉTODOS DE DETERMINAÇÃO DE CARBONO ORGÂNICO TOTAL NO SOLO ${ }^{(1)}$
}

\author{
Danilo dos Santos Rheinheimer ${ }^{(2)}$, Ben-Hur Costa de Campos $^{(3)}$, \\ Sandro José Giacomini ${ }^{(2)}$, Paulo Cesar Conceição ${ }^{(4)}$ \& Edson \\ Campanhola Bortoluzzi ${ }^{(5)}$
}

\begin{abstract}
RESUMO
O teor de C orgânico total do solo (COT) pode ser determinado por métodos que se baseiam nos princípios de combustão a seco e combustão úmida. Ambos apresentam inconvenientes, principalmente o de combustão úmida, que exige grande quantidade de reagentes, gerando, por conseqüência, alta quantidade de resíduos tóxicos que contêm Cr. O método denominado Mebius no bloco de digestão (Mebius no bloco) permite a diminuição do uso de dicromato de potássio em análises de solo. Nesse sentido, o presente estudo objetivou verificar a precisão e exatidão do método Mebius no bloco em relação a outros de combustão úmida e o de combustão a seco. O trabalho foi realizado na Universidade Federal de Santa Maria, em duas etapas: os teores de COT foram determinados em 18 amostras de duas camadas (0-5 e 5-10 cm) de um Latossolo Vermelho distrófico típico com diferentes usos. Os métodos usados foram Walkley-Black, Mebius modificado, Mebius no bloco e por captura de $\mathrm{CO}_{2}$; os teores de COT foram determinados por combustão a seco e Mebius no bloco em 75 amostras coletadas nos horizontes A1 (0-7,5 e 7,5-15 cm), A2, E e Bt de um Argissolo Vermelho-Amarelo distrófico abrúptico submetido a cinco sistemas de manejo e três repetições. A precisão apresentada pelos métodos de combustão úmida é similar e com coeficiente de variação abaixo de $10 \%$, com exceção do método de captura de $\mathrm{CO}_{2}$, que apresentou valores de COT inferiores aos dos demais e com maior coeficiente de variação. $O$ método Mebius no bloco permite processar maior número de amostras por tempo,
\end{abstract}

(1) Recebido para publicação em fevereiro de 2007 e aprovado em agosto de 2007.

${ }^{(2)}$ Professor do Departamento de Solos, Centro de Ciências Rurais, Universidade Federal de Santa Maria - UFSM. Camobi, CEP 97105-900 Santa Maria (RS). E-mails: danilor@smail.ufsm.br; sjgiacomini@smail.ufsm.br

(3) Doutorando do Programa de Pós-graduação em Ciência do Solo, UFSM.

(4) Doutorando do Programa de Pós-graduação em Ciência do Solo, Universidade Federal do Rio Grande do Sul - UFRGS. Av. Bento Gonçalves 7712, CEP 90001-970 Porto Alegre (RS).

${ }^{(5)}$ Professor Pesquisador Adjunto I da Faculdade de Agronomia e Medicina Veterinária da Universidade de Passo Fundo - UPF. Caixa Postal 611, CEP 99001-970 Passo Fundo (RS). E-mail: edsonb@upf.br 
com menor consumo de reagentes, e seus resultados apresentam boa precisão (coeficiente de variação menor que 2,60 \%) entre os métodos de combustão úmida testados. Um fator de correção de 1,14 deverá ser aplicado aos resultados obtidos pelo método Mebius no bloco para equivaler aos obtidos por combustão a seco (analisador elementar de carbono).

Termos de indexação: Mebius no bloco digestor, precisão e exatidão, dicromato de potássio, resíduos químicos.

\title{
SUMMARY: COMPARISON OF DETERMINATION METHODS OF TOTAL ORGANIC CARBON INSOILS
}

\begin{abstract}
The total organic carbon (TOC) in soils can be estimated by dry and humid combustion methods. Both have some drawbacks, mainly the humid combustion, which demands high reagent quantities and generates toxic waste containing chromium. The Mebius in Block method was developed to decrease the quantity of $\mathrm{Na}$ dichromate used in analyses. The objectives of this study were to verify the precision and accuracy of the Mebius in Block compared with other wet and dry combustion methods. The study was performed in two phases at the Universidade Federal de Santa Maria: (a) TOC contents of 18 soil samples of a Rhodic Ferralsol were determined in the $0-5$ and 5-10 cm layers, by different practices. The methods evaluated were Walkley-Black, modified Mebius, Mebius in digestion block, and by $\mathrm{CO}_{2}$ capturing; (b) The TOC contents were determined by Mebius in Block and an elementary analyzer of carbon and nitrogen in 75 samples collected in A1 (0-7.5 and 7.5-15 cm layers), A2, $E$ and Bt horizons of a Rhodic Acrisol with five management practices and three replicates. The data precision of the humid combustion methods was similar, with a coefficient of variation $(\mathrm{CV})$ below $10 \%$, except for the $\mathrm{CO}_{2}$ capturing method, where the TOC contents were lower, while the variation coefficient was higher. The Mebiusin-block processes a larger number of samples with lower reagent consumption and a greater precision $(C V<2.60 \%)$ than the other methods. A factor of correction of 1.14 should be applied to Mebius-in-Block values for an adjustment to those obtained by dry combustion (elementary analyzer).
\end{abstract}

Index terms: Mebius in the digestion block, potassium dichromate, precision and accuracy, toxic waste.

\section{INTRODUÇÃO}

De modo geral, considera-se que a matéria orgânica do solo contém cerca de $58 \%$ de $\mathrm{C}$, em relação à massa total. Assim, a determinação do carbono orgânico total (COT) tem sido utilizada para estimar quantitativamente a fração orgânica do solo (Nelson \& Sommers, 1982), o que auxilia no entendimento de suas várias propriedades químicas, físicas e biológicas. Atualmente, existem vários métodos para se determinar o $\mathrm{C}$ em amostras de solo. O método da combustão a seco é considerado padrão, devido a sua alta precisão e exatidão nos resultados (Soon \& Abboud, 1991; Nelson \& Sommers, 1996). Neste método, a amostra é oxidada em presença de $\mathrm{Cr}_{2} \mathrm{O}_{7}{ }^{2-} \mathrm{e} \mathrm{CO}_{3} \mathrm{O}_{4} / \mathrm{Ag}$ em forno, à temperatura de $1.020-1.500{ }^{\circ} \mathrm{C}$, alimentado com $\mathrm{O}_{2}$ sob pressão de $30 \mathrm{kPa}$. As moléculas orgânicas são oxidadas na forma $\mathrm{N}_{\mathrm{x}} \mathrm{O}_{\mathrm{y}}$ e $\mathrm{CO}_{2^{-}}$ $\mathrm{H}_{2} \mathrm{O}+\mathrm{O}_{2}$, que são carregadas, nas formas de gás, para um forno de redução, sob fluxo de hélio. O forno de redução contém íons $\mathrm{Cu}$, à temperatura de $750{ }^{\circ} \mathrm{C}$, de onde o $\mathrm{N}$ sai na forma de $\mathrm{N}_{2}$. Posteriormente, $\mathrm{o} \mathrm{N}_{2} \mathrm{e}$ o $\mathrm{CO}_{2}$ são separados em uma coluna cromatográfica e determinados em detector apropriado. Enquanto os laboratórios de rotina no Brasil não possuírem equipamentos que dosem o $\mathrm{C}$ do solo por combustão a seco, justifica-se a busca de métodos alternativos que utilizem menor quantidade de reagentes tóxicos, com boa precisão e exatidão.

Os métodos de combustão úmida são mais acessíveis, apesar de utilizarem reagentes muito tóxicos (Rodella \& Alcarde, 1994; Pimentel et al., 2006). Esses métodos baseiam-se na redução do dicromato $\left(\mathrm{Cr}_{2} \mathrm{O}_{7}{ }^{2-}\right)$ por compostos de $\mathrm{C}$ orgânico e subseqüente determinação do $\mathrm{Cr}_{2} \mathrm{O}_{7}{ }^{2-}$ não reduzido por titulação de oxirredução com $\mathrm{Fe}^{2+}$ ou por técnicas colorimétricas (Nelson \& Sommers, 1996). Os métodos propostos por Walkley-Black (Nelson \& Sommers, 1982; Tedesco et al., 1995) e Mebius modificado (Nelson \& Sommers, 1982) baseiam-se também nesse princípio. Contudo, o método Walkley \& Black (1960), descrito por Nelson \& Sommers (1982), originalmente subtrai o aquecimento da amostra, o que, segundo os autores, compromete a exatidão dos 
resultados. Nesse sentido, Tedesco et al. (1995) propuseram a introdução de uma rotina de padronização, em que as amostras são aquecidas a $150{ }^{\circ} \mathrm{C}$ por $1 \mathrm{~min}$. Essa modificação melhorou a exatidão dos resultados, mas dificultou a operacionalidade na determinação do COT, pois as amostras podem não ser aquecidas igualmente, o que afeta a homogeneidade do conjunto e, conseqüentemente, a precisão do método. No entanto, este método foi o precursor dos métodos usados em análises do solo no Brasil.

O método proposto por Allison (1960), citado por Nelson \& Sommers (1982), utiliza o bloco de digestão. O princípio baseia-se na captura do $\mathrm{CO}_{2}$ evoluído, com uma base forte, titulando-se a alcalinidade excedente. Assim, obtém-se unicamente o COT do solo, porém não foram eliminados os inconvenientes apresentados no método de combustão a seco (alto custo) e no método de combustão a úmido (operacionalidade reduzida). Nessas condições, há dúvidas quanto à eficiência da evolução e captação do $\mathrm{CO}_{2}$, que pode subestimar o teor de COT e, por conseqüência, a de matéria orgânica do solo.

Yeomans \& Bremner (1988) propuseram o uso do bloco de digestão (Mebius no bloco), no intuito de uniformizar a temperatura durante o processo de digestão, aumentando o número de amostras manipuladas por bateria. Esse método permitiu também diminuir a quantidade de solo e de dicromato utilizados. Contudo, resta saber se a precisão desse método é compatível com aquela dos métodos de combustão úmida utilizados em geral nos laboratórios de análise de solo e se a exatidão é compatível com os padrões oferecidos pelo método de combustão a seco. Dessa forma, o presente trabalho foi desenvolvido com o objetivo de avaliar a precisão de diferentes métodos de determinação de COT pela combustão úmida, bem como a eficiência do método Mebius no bloco na recuperação de $\mathrm{C}$ orgânico em amostras de solo, em relação ao de combustão a seco.

\section{MATERIAL E MÉTODOS}

O trabalho foi realizado no Departamento de Solos da Universidade Federal de Santa Maria, em duas etapas. Na primeira, foram comparados os principais métodos de digestão úmida, a saber: Walkley-Black modificado por Tedesco et al. (1995), sem fator de correção, captura de $\mathrm{CO}_{2}$ descrito por Nelson \& Sommers (1982), Mebius modificado por Nelson \& Sommers (1982) e Mebius adaptado para bloco de digestão(Mebius no bloco) descrito por Yeomans \& Bremner (1988). Este último é descrito a seguir, em detalhes.

Amostras de terra fina seca ao ar (TFSA), de 0,20 a $0,50 \mathrm{~g}$, foram colocadas em tubos de vidro de $2,5 \mathrm{~cm}$ de diâmetro e $25,0 \mathrm{~cm}$ de altura. A solução oxidante composta de $10 \mathrm{~mL}$ de $\mathrm{K}_{2} \mathrm{Cr}_{2} \mathrm{O}_{7} 0,067 \mathrm{~mol} \mathrm{~L}^{-1}$ mais
$15 \mathrm{~mL}$ de $\mathrm{H}_{2} \mathrm{SO}_{4}$ concentrado foi adicionada lentamente pela parede do tubo. Os tubos foram colocados em bloco de digestão, juntamente com uma esfera de vidro posicionada na extremidade superior do tubo, para servir de condensador. Em cada bateria de 40 amostras utilizaram-se três provas em branco aquecidas e três provas em branco sem aquecimento, para o momento em que a análise foi realizada. Após o pré-aquecimento do bloco a $150{ }^{\circ} \mathrm{C}$, os tubos foram colocados no bloco, permanecendo sob aquecimento por 30 min. O conteúdo dos tubos foi transferido para erlenmeyer, juntamente com $80 \mathrm{~mL}$ de água destilada. As amostras foram então tituladas com sulfato ferroso amoniacal $\left[\mathrm{Fe}\left(\mathrm{NH}_{4}\right)_{2}\left(\mathrm{SO}_{4}\right)_{2} \cdot 6 \mathrm{H}_{2} \mathrm{O}\right]$ $0,2 \mathrm{~mol} \mathrm{~L}^{-1}$, na presença de cinco gotas do indicador ferroína (O-fenantrolina), até o aparecimento da cor violeta. A concentração real do sulfato ferroso amoniacal foi determinada por titulação de provas em branco sem aquecimento. Algumas provas em branco também foram submetidas ao aquecimento, para controlar uma possível redução do dicromato durante o processo.

As amostras de solo foram coletadas em triplicata nas camadas de $0-5$ e $5-10 \mathrm{~cm}$ de um Latossolo Vermelho distrófico típico (Embrapa, 1999) sob mata nativa, campo nativo e sistema de plantio direto, no município de Cruz Alta (RS). As amostras foram secas ao ar e peneiradas em malha de $2,0 \mathrm{~mm}$. Após secagem, elas foram cuidadosamente separadas dos resíduos de raízes, moídas em pilão e almofariz e novamente peneiradas em malha de $1,0 \mathrm{~mm}$. Essas amostras apresentaram variação nos teores de argila de 352 a $425 \mathrm{~g} \mathrm{~kg}^{-1}$.

A segunda etapa constou do ajuste de uma equação de regressão, que relacionou os teores de C orgânico total obtidos pelo método da digestão úmida Mebius no bloco com aqueles obtidos pelo método de combustão a seco. Para isso, foram utilizadas 75 amostras de um Argissolo Vermelho-Amarelo distrófico abrúptico (Embrapa, 1999), provenientes de experimento em Santa Maria (RS). As amostras foram coletadas em cinco camadas (A1 0-7,5 cm, A1 7,5-15 cm, A2, E e $\mathrm{Bt}$ ), com três repetições a campo, e sob cinco usos (sistema plantio direto a partir de campo nativo e a partir do cultivo convencional, campo nativo, cultivo convencional e mata de pinus). Essas amostras apresentaram grande amplitude de teores de argila (de 95 a $468 \mathrm{~g} \mathrm{~kg}^{-1}$ ), C orgânico (de 2 a $40 \mathrm{~g} \mathrm{~kg}^{-1}$ ) e CTC (de 4 a $14 \mathrm{cmol}_{\mathrm{c}} \mathrm{kg}^{-1}$ ). Determinou-se o teor de C orgânico total pelo método do bloco, descrito na etapa anterior, e pela combustão a seco, utilizando um analisador elementar de C e N. Para determinação de C orgânico total, as análises de laboratório foram realizadas em duas repetições. Os dados foram ajustados por uma regressão linear, tomando-se os valores de $\mathrm{C}$ obtidos pelo método de combustão a seco como variável independente. Um fator de correção dos teores de COT para o método do Mebius no bloco foi obtido por ajuste de uma nova equação com interseção na origem. 


\section{RESULTADOS E DISCUSSÃO}

Todos os métodos mostraram-se sensíveis, refletindo as variações naturais dos teores de COT decorrentes das diferentes condições de uso e da camada de amostragem (Quadro 1). A precisão dos métodos na determinação de COT do solo foi alta, tendo em vista os coeficientes de variação inferiores a $10 \%$.

$\mathrm{O}$ método de captura de $\mathrm{CO}_{2}$, comparado aos demais, apresentou teores inferiores de COT em todas as condições estudadas, exceto para as amostras de mata nativa na camada de $5-10 \mathrm{~cm}$ (Quadro 1). Este método apresentou coeficientes de variação entre 1,50 e $8,20 \%$, que, mesmo sendo considerados baixos, foram superiores aos dos demais métodos, principalmente para as amostras da camada superficial dos solos (Quadro 1). Embora este método apresente boa nitidez do ponto de viragem, de rosa cintilante para branco ou incolor, no processo de titulação, mostrou o inconveniente da formação de uma camada de material no fundo de alguns tubos. Supõe- se que esse inconveniente possa ter influenciado a precisão dos resultados, refletindo-se em coeficientes de variação mais elevados.

O método Walkley-Black mostrou coeficientes de variação inferiores a 1,30 \% nas amostras superficiais (0-5 cm) da mata e campo nativo, com valores elevados de COT (Quadro 1). Contudo, apresentou coeficientes de variação superiores a $1,80 \%$ nas amostras da camada de $5-10 \mathrm{~cm}$, com valores baixos de COT. Esses resultados sugerem que o método Walkley-Black pode ser mais preciso para teores mais elevados de COT.

Os teores de COT obtidos pelo método Mebius no bloco não diferiram daqueles obtidos pelos métodos Walkley-Black e Mebius modificado. No entanto, os coeficientes de variação obtidos ficaram sempre abaixo de $2,6 \%$, sendo menores em relação aos demais métodos para a maioria das situações estudadas. A padronização do aquecimento das amostras no bloco de digestão pode ter diminuído a interferência do operador, influenciando os baixos valores do coeficiente

Quadro 1. Teor de C orgânico total (COT), de 18 amostras de um solo Latossolo Vermelho, determinado por diferentes métodos de combustão úmida oriundos de três sistemas de uso do solo e duas profundidades. Santa Maria (RS), 2006

\begin{tabular}{|c|c|c|c|c|}
\hline \multirow{2}{*}{ Uso do solo } & \multicolumn{4}{|c|}{ Métodos de análise de carbono por combustão úmida } \\
\hline & Walkley-Black & Mebius modificado & Mebius no bloco & Captura de $\mathrm{CO}_{2}$ \\
\hline \multicolumn{5}{|l|}{ Mata nativa } \\
\hline Média COT, $\mathrm{g} \mathrm{kg}^{-1}$ & $35,01 \mathrm{a}^{(1)}$ & $35,32 \mathrm{a}$ & $34,68 \mathrm{a}$ & $31,50 \mathrm{~b}$ \\
\hline Desvio-padrão & 0,44 & 0,80 & 0,46 & 0,78 \\
\hline $\mathrm{CV}(\%)$ & 1,30 & 2,30 & 1,30 & 2,50 \\
\hline \multicolumn{5}{|l|}{ Campo nativo } \\
\hline Média COT, $\mathrm{g} \mathrm{kg}^{-1}$ & $27,44 \mathrm{ab}$ & $28,46 \mathrm{a}$ & $28,00 \mathrm{a}$ & $26,37 \mathrm{~b}$ \\
\hline Desvio-padrão & 0,15 & 0,36 & 0,72 & 1,19 \\
\hline $\mathrm{CV}(\%)$ & 0,60 & 1,30 & 2,60 & 4,50 \\
\hline \multicolumn{5}{|l|}{ Plantio direto } \\
\hline Média COT, $\mathrm{g} \mathrm{kg}^{-1}$ & $25,02 \mathrm{a}$ & $25,82 \mathrm{a}$ & $25,83 \mathrm{a}$ & $23,28 b$ \\
\hline Desvio-padrão & 0,63 & 0,28 & 0,35 & 1,14 \\
\hline $\mathrm{CV}(\%)$ & 2,50 & 1,10 & 1,34 & 4,90 \\
\hline \multicolumn{5}{|l|}{ Mata nativa } \\
\hline Média COT, $\mathrm{g} \mathrm{kg}^{-1}$ & $19,69 \mathrm{a}$ & $18,27 \mathrm{~b}$ & $18,68 \mathrm{ab}$ & $17,49 \mathrm{~b}$ \\
\hline Desvio-padrão & 1,08 & 1,18 & 0,20 & 0,26 \\
\hline $\mathrm{CV}(\%)$ & 5,50 & 6,50 & 1,10 & 1,50 \\
\hline \multicolumn{5}{|l|}{ Campo nativo } \\
\hline Média COT, $\mathrm{g} \mathrm{kg}^{-1}$ & $21,48 \mathrm{a}$ & $23,22 \mathrm{a}$ & $22,47 \mathrm{a}$ & $19,75 b$ \\
\hline Desvio-padrão & 1,12 & 0,35 & 0,42 & 1,61 \\
\hline $\mathrm{CV}(\%)$ & 5,20 & 1,50 & 1,90 & 8,20 \\
\hline \multicolumn{5}{|l|}{ Plantio direto } \\
\hline Média COT, $\mathrm{g} \mathrm{kg}^{-1}$ & $18,40 \mathrm{a}$ & $18,07 \mathrm{a}$ & $18,58 \mathrm{a}$ & $16,65 \mathrm{c}$ \\
\hline Desvio-padrão & 0,33 & 0,73 & 0,15 & 0,76 \\
\hline $\mathrm{CV}(\%)$ & 1,80 & 4,00 & 0,80 & 4,60 \\
\hline
\end{tabular}

(1) Médias seguidas pela mesma letra minúscula nas linhas não diferem entre si pelo teste de DMS (mínima diferença significativa) com t de Student a $5 \%$. 
de variação - inconveniente observado no método de Walkley-Black. Nesse sentido, estima-se que o aquecimento das amostras no bloco de digestão proporcionou, em geral, uma precisão, no mínimo, similar à do método Walkley-Black na determinação do COT, permitindo, além disso, aumentar o número de amostras analisadas, em relação aos outros métodos.

Para que o método Mebius no bloco possa ser empregado em laboratórios de rotina e de pesquisa, é necessário correlacioná-lo com o método-padrão (combustão a seco) para avaliar a sua exatidão. Obteve-se alta correlação entre os teores de COT determinados pelo método da combustão a seco e pelo método de digestão úmida do Mebius no bloco. A dispersão dos pontos em torno da reta foi baixa, resultando coeficiente de determinação elevado $\left(\mathrm{R}^{2}=0,97\right)$. O ajuste matemático foi expresso pela equação $\hat{y}=-0,9770+0,9584 x$, em que $(\hat{y})$ é o teor de COT estimado pelo método Mebius no bloco e (x) o teor de COT determinado pelo método de combustão a seco, ambos expressos em $\mathrm{g} \mathrm{kg}^{-1}$ de COT (Figura 1). Observou-se que, quando o teor de $\mathrm{C}$ orgânico total era zero, determinado pela combustão a seco, o valor estimado pela combustão úmida era de $-0,977 \mathrm{~g} \mathrm{~kg}^{-1} \mathrm{e}$ a inclinação da reta, muito próxima de $1(0,958)$. Desse modo, forçando a passagem da reta de regressão pela origem, obteve-se a equação $\hat{y}=0,878 x$, cujo fator de correção dos teores de COT obtidos pelo Mebius no bloco é de 1,14. Este valor é muito próximo do obtido por Tedesco et al. (1995), de 1,12, para o método de Walkley-Black.

O Mebius no bloco subestima os valores de C orgânico total, comparado à combustão a seco, refletindo-se então na exatidão do método. Isso pode ser devido às associações organominerais que protegem

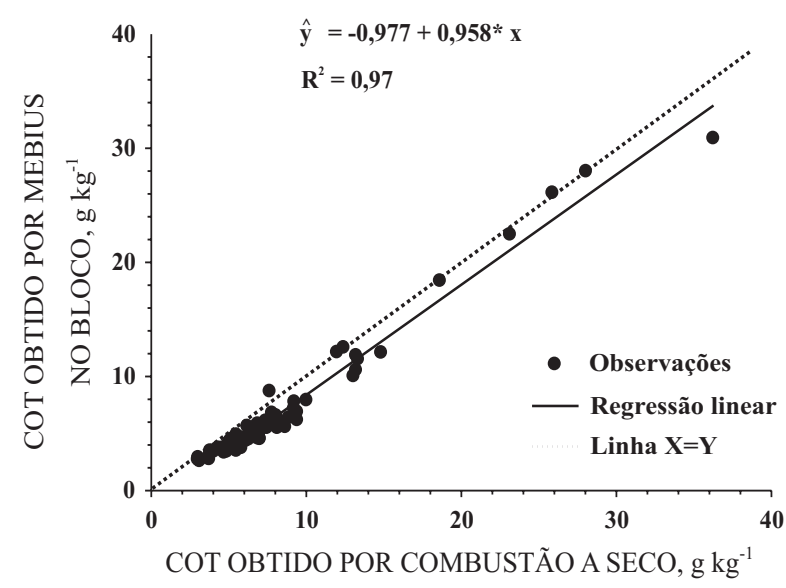

Figura 1. Regressão linear entre os teores de C orgânico total (COT) de um Argissolo VermelhoAmarelo distrófico abrúptico estimados pelos métodos da combustão a seco e Mebius no bloco, Santa Maria, RS, 2006. de forma física e química a matéria orgânica no seio de microagregados de partículas de argila (Chenu, 2001). Não obstante a necessidade de aplicação de fator de correção dos valores obtidos, acredita-se que a adoção do método Mebius no bloco pode trazer vantagens operacionais nos laboratórios de rotina de análises de solo do Brasil. A manipulação de maior número de amostras e o maior controle do viés do operador na etapa de elevação da temperatura das amostras são exemplos disso. Ademais, este método mantém a eficiência na determinação de COT obtida por outros métodos, como o Walkley-Black. Adicionalmente, há perspectiva de diminuição do uso de reagentes com elementos tóxicos, como o $\mathrm{Cr}$, com vantagens óbvias no tratamento e manipulação de efluentes dos laboratórios de análise de solos. Esse fato é importante porque o bicromato pode ser muito tóxico quando inalado ou ingerido, podendo provocar câncer e mesmo alterações genéticas hereditárias, além de provocar sensibilização em contato com a pele e vias respiratórias (Pimentel et al., 2006). Quando não é devidamente recuperado após uso em laboratórios, esse reagente pode ser muito tóxico para organismos aquáticos, apresentando $\mathrm{DL}_{50}$ de $25 \mathrm{mg} \mathrm{L}^{-1}$. Tendo em vista o exposto, sugere-se que os laboratórios busquem sistemas de tratamento de resíduos eficientes, bem como alternativas de análises que possibilitem ao mesmo tempo diminuir o uso de reagentes sem que isso interfira negativamente na qualidade da determinação de $\mathrm{C}$ orgânico total.

\section{CONCLUSÕES}

1. O método da captura de $\mathrm{CO}_{2}$ apresenta menor precisão e exatidão com subestimação significativa dos teores de C orgânico total do solo, comparado aos demais métodos de combustão úmida.

2. O método Mebius no bloco apresenta tendência de maior precisão em relação aos métodos WalkleyBlack e Mebius modificado na determinação de C orgânico total em solos com condições variadas de uso, porém subestima os valores em relação ao métodopadrão.

3. O fator de correção que deverá ser aplicado aos resultados obtidos pelo método Mebius no bloco para equivaler aos teores obtidos pelo analisador elementar de $\mathrm{C}$ é de 1,14 .

\section{LITERATURA CITADA}

CHENU, C. Le complexe argilo-humique des sols: Etat des connaissances actuelles. C. R. Acad. Agric. France, 87:3$12,2001$.

EMPRESA BRASILEIRA DE PESQUISA AGROPECUÁRIA EMBRAPA. Sistema brasileiro de classificação de solos. Rio de Janeiro, 1999. 412p. (Documentos, 15) 
NELSON, D.W. \& SOMMERS, L.E. Total carbon, organic carbon and organic matter. In: PAGE, A.L.; MILLER, R.H. \& KEENEY, D.R., eds. Methods of soil analysis: Chemical and microbiological properties. Part 2. Madison, Soil Science Society of America, 1982. p.539-579.

NELSON, D.W. \& SOMMERS, L.E. Total carbon, organic carbon and organic matter. In: SPARKS, D.L.; PAGA, A.L.; HELMKE, P.A.; LOEPPERT, R.H.; SOLTANPOUR, P.N.; TABATABAI, M.A.; JOHNSTON, C.T. \& SUMMER, M.E., eds. Methods of soil analysis: Chemical methods. Part 3. Madison, Soil Science Society of America, 1996. p.961-1010.

PIMENTEL, L.C.F.; CHAVES, C.R.; FREIRE, L.A.A. \& AFONSO, J.C. O inacreditável emprego de produtos químicos perigosos no passado. Química Nova, 29:1138-1149, 2006.
RODELLA, A. \& ALCARDE, J.C. Avaliação de materiais orgânicos empregados como fertilizantes. Sci. Agric., 51:556-562, 1994.

SOON, Y.K. \& ABBOUD, S. A comparison of some methods for soil organic carbon determination. Comm. Soil Sci. Plant Anal., 22:943-954, 1991.

TEDESCO, M.J.; GIANELLO, C.; BISSANI, C.A.; BOHNEN, H. \& WOLKWEISS, S.J. Análises de solo, plantas e outros materiais. 2.ed. Porto Alegre, Universidade Federal do Rio Grande do Sul, 1995. 174p. (Boletim Técnico, 5)

YEOMANS, J.C. \& BREMNER, J.M. A rapid and precise method for routine determination of organic carbon in soil. Comm. Soil Sci. Plant Anal., 19:1467-1476, 1988. 